\title{
Tendências temporais da prática de atividade física no lazer de adultos na cidade de São Luís - MA, Brasil (2006-2018)
}

\author{
Dartel Ferrari de Lima \\ Professor da Universidade Estadual do Oeste do Paraná, doutor em Medicina Preventiva (FMUSP), orientador \\ permanente do Programa de Pós-Graduação em Educação em Ciência e Educação Matemática \\ $\square$ dartelferrari07@gmail.com
}

Laize Biesdorf Winter

Graduada em Comunicação Social - Publicidade e Propaganda, membro do Grupo de Estudo em Educação Física e Saúde da Universidade Estadual do Oeste do Paraná

$\triangle$ wlaize@gmail.com

Lauana de Lima Tosti

Membro do Grupo de Estudo em Educação Física e Saúde da Universidade Estadual do Oeste do Paraná $\triangle$ lautosti.15@gmail.com

Vitor Henrique Souza Santana

Membro do Grupo de Estudo em Educação Física e Saúde da Universidade Estadual do Oeste do Paraná $\triangle$ vhsantana2018@gmail.com

\section{Lohran Anguera Lima}

Médico da Santa Casa de Misericórdia de São Paulo, especialista em Ortopedia e Traumatologia, Membro da Sociedade Brasileira de Ortopedia e Traumatologia (SBOT), da North American Spine Society (NASS) e da AOSpine

$\bowtie$ lohranangueralima@gmail.com

\section{Resumo:}

O objetivo deste estudo foi analisar as tendências temporais dos principais tipos de atividade física (AF) praticados no lazer na cidade de São Luís (MA), Brasil, no período de 2006 a 2018. Foram analisados dados de 24.412 adultos de ambos os sexos ( $\geq 18$ anos de idade), participantes do Sistema de Vigilância de Fatores de Risco e Proteção para Doenças Crônicas por Inquérito Telefônico (Vigitel). As tendências temporais foram analisadas para o indicador: principal modalidade de exercício físico e esporte praticado no lazer. Para os cinco tipos de atividades mais citados, a caminhada foi preferida por ambos os sexos, com $20,3 \%$ e $17,7 \%$ da população feminina e masculina, respectivamente, resultando tendência de crescimento de 5,5 pontos percentuais a favor dos homens quando comparados às mulheres. $O$ futebol apresentou predominância masculina (17,3\%) com tendência de decréscimo para o período (-10,7\%). A musculação foi a segunda preferência para as mulheres $(5,4 \%)$ e a terceira para os homens $(7,2 \%)$, com tendência estabilizada para ambos os sexos. Para cada quatro ciclistas de São Luís, três deles eram homens e não foi observada tendência de crescimento. Contrariamente, houve tendência de crescimento para a corrida (entre homens) e para a ginástica e hidroginástica (entre mulheres). Políticas baseadas na melhoria da infraestrutura e segurança individual podem contribuir para reduzir as diferenças dos percentuais de AF no lazer entres os sexos. A continuidade e a periodicidade do sistema de monitoramento da AF pelo Vigitel permitirá embasar ações promotoras da AF a partir de mudanças ao longo do tempo.

Palavras-chave: Atividade motora, Vigilância em saúde pública, Vigilância populacional, Epidemiologia. 


\title{
Trends in leisure-time physical activity among adults in the city of São Luís - MA, Brazil (2006-2018)
}

\begin{abstract}
:
The aim of the present study was to evaluate time trends of the main types exercise or sport practiced during leisure time from 2006 to 2018 among adults living in São Luís (MA), Brazil. We analyzed data from 24,412 adults ( $\geq 18$ years old) participants of the Surveillance System on Risk and Protective Factors for Chronic Disease through Telephone Interviews (Vigitel). Time trends of the following indicators were evaluated leisure-time. The walking was the most prevalent modality of exercise in both sexes, with $20.3 \%$ and $17.7 \%$ of the female and male population respectively, and a trend of average growth of 5.5\% higher in men compared to women. The recreational football had a male predominance (17.3\%) with negative growth (-10.7\%). Bodybuilding was the second preference for leisure (PA) for women (5.4\%) and the third for man (7.2\%), with the stabilized variation pattern for both sexes. For every four cyclists riding in São Luís, three of them are men. Running (men) and gymnastics and water aerobics (women), growing, completed the preferences. Policies based on improved infrastructure and individual security can contribute to reducing gender differences in leisure-time PA. The continuity and periodicity of Vigitel's PA monitoring system will support actions promoting PA based on changes observed over time.

Keywords: Motor activity, Public health surveillance, Population surveillance, Epidemiology.
\end{abstract}

\section{Tendencias temporales de la actividad física en el tempo libre en adultos en São Luís - MA, Brasil (2006-2018)}

\begin{abstract}
Resumen:
El objetivo del estudio fue analizar las tendencias temporales de los principales tipos de AF en el tiempo libre en la ciudad de São Luís (MA), Brasil, de 2006 a 2018. Se analizaron datos de 24,412 adultos de ambos sexos ( $\geq 18$ años), participantes en el Sistema de vigilancia de factores y riesgos de la encuesta telefónica (Vigitel). Se analizaron las tendencias temporales para el indicador: modalidad principal de ejercicio físico y deporte practicado en el tiempo libre. Para las cinco modalidades más citadas, se prefirió caminar para ambos sexos, con $20.3 \%$ y $17.7 \%$ de practicantes en la población femenina y masculina, respectivamente, y una tendencia de crecimiento $5.5 \%$ mayor en los hombres en comparación. las mujeres. El fútbol mostró predominio masculino (17.3\%) con un patrón de disminución para el período (-10.7\%). El culturismo fue la segunda preferencia para las mujeres (5.4\%) y la tercera para los hombres (7.2\%) con el patrón de variación estabilizada para ambos sexos. Por cada cuatro ciclistas que practican ciclismo en São Luís, tres de ellos son hombres que mantienen constante el patrón de variación. Correr (hombres) y la creciente gimnasia y aeróbicos acuáticos (mujeres) completaron las preferencias. Las políticas basadas en la mejora de la infraestructura y la seguridad individual pueden contribuir a reducir las diferencias de género en la AF de tiempo libre. La continuidad y periodicidad del sistema de monitoreo de AF de Vigitel respaldará acciones que promuevan la $\mathrm{AF}$ en función de los cambios observados a lo largo del tiempo.

Palabras clave: Actividad motora, Vigilancia en salud pública, Vigilancia poblacional, Epidemiología.
\end{abstract}

\section{INTRODUÇÃo}

Os benefícios da atividade física (AF) para a manutenção da saúde física e mental estão robustamente demonstrados na literatura (KOHL et al., 2012; WHO, 2013). No entanto, é 
relativamente recente a preocupação com a inatividade física pelos serviços de atenção primária da saúde pública (WHO, 2010). Essa preocupação, crescente, se deve pela associação positiva da inatividade física com as doenças crônicas não transmissíveis (WHO, 2013). Observa-se que a vida contemporânea, de modo geral, as novas tecnologias requerem níveis menores de $\mathrm{AF}$ do que no passado. Há evidências robustas de que nos últimos 40 anos, o nível de AF da população caiu acentuadamente nos países de maior renda per capita, com a expectativa de que os países de média e baixa renda sigam essa tendência (HALLAL et al., 2012; GUTHOLD et al., 2018).

No ano de 2018, estimativas de prevalência de AF insuficiente - abaixo de 150 minutos de AF de intensidade moderada, ou 75 minutos de intensidade vigorosa por semana, ou qualquer combinação equivalente dos dois (WHO, 2010) - envolvendo 1,9 milhões de participantes adultos ( $\geq 18$ anos de idade), recrutados em 168 países de cinco continentes (Américas, Europa, Ásia, Oceania e África), mostrou taxa global de AF insuficiente de 27,5\% (IC95\%: 25,0-32,2), variando de 5,5\% na Uganda (África Subsaariana) a 67,0\% no Kuwait (Ásia Ocidental) (GUTHOLD et al., 2018). O Brasil apresentou taxa de AF insuficiente de 47,0\% (IC95\%: 38,9-55,3), se posicionando acima da média para a América Latina e Caribe (39,1\%; IC95\%: 37,8-40,6). No mesmo ano (2018), o estudo nacional que monitora a prática de AF de brasileiros adultos variou de 36,1\% no Distrito Federal e 50,6\% em João Pessoa. Entre os homens, as maiores frequências foram encontradas em Teresina (41,2\%), Fortaleza $(41,1 \%)$ e João Pessoa $(41,0 \%)$ e, entre as mulheres, as maiores frequências foram encontradas em João Pessoa (58,6\%), Recife $(58,3 \%)$ e Fortaleza (58,1\%) (BRASIL, 2018).

A promoção da saúde mediada por mudanças para o estilo de vida mais ativo é destaque no Plano de Ação Global para a prevenção e controle de doenças crônicas não transmissíveis 2013-2020 da Organização Mundial da Saúde (OMS), onde prevê a redução de 10\% de pessoas fisicamente inativas até o ano de 2025 (WHO, 2019). O progresso para alcançar essa meta da OMS, necessita de ações aceleradas para inverter as tendências globais e brasileiras. Políticas e programas de incentivo à participação em AF são necessários para alterar os níveis de inatividade física em localidades onde as taxas são elevadas. Espera-se que a implementação de intervenções direcionadas e baseadas em evidências possam ampliar os efeitos direcionados à saúde da população pela promoção da AF. Desse modo, este estudo teve como objetivo fornecer informações sobre a série histórica (2006 a 2018) dos tipos principais 
de exercício físico e esporte recreativo praticados por adultos residentes na cidade de São Luís -MA, Brasil, na intenção de promover informações pontuais aos profissionais responsáveis pela promoção da saúde populacional de São Luiz mediada pelo incentivo à prática de AF.

\section{MÉTODOLOGIA}

A cidade de São Luís é a capital do estado do Maranhão, que juntamente com mais oito estados, constituem a região Nordeste do Brasil. o índice de desenvolvimento humano de São Luís é de 0,768 , classificado como alto índice de desenvolvimento humano, ficando acima da média da região Nordeste $(0,608)$. A população de São Luís, para o ano de 2018, foi estimada em 1.094.667 habitantes, sendo a cidade mais populosa do estado do Maranhão, a quarta da região Nordeste e a $15^{\mathrm{a}}$ do país. Em São Luís, residem mais mulheres do que homens. A população é predominante feminina, composta de $53,2 \%$ de mulheres e $46,8 \%$ de homens. A população adulta adultos ( $\geq 18$ anos de idade) da capital maranhense foi estimada em $81,8 \%$, sendo $94 \%$ da população residentes da zona urbana do município (IBGE, 2017).

Neste estudo, foram examinados dados transversais do sistema de Vigilância de Fatores de Risco e Proteção para Doenças Crônicas não Transmissíveis por Inquérito Telefônico (Vigitel) de 2006 a 2018, referentes à cidade de São Luís, cuja metodologia própria pode ser acessada em publicação prévia (BRASIL, 2016). Os procedimentos de amostragem empregados pelo Vigitel visam obter amostras da população de adultos residentes em domicílios servidos por linha telefônica fixa em cada uma das capitais dos estados brasileiros e do Distrito Federal. O sistema Vigitel estabelece um tamanho amostral mínimo de aproximadamente dois mil participantes em cada cidade para estimar com erro máximo de cerca de dois pontos percentuais a frequência de qualquer fator de risco na população adulta. De modo adicional, amostras menores, são aceitas nas localidades em que a cobertura de telefonia fixa seja inferior a $40 \%$ dos domicílios e, que o número absoluto de domicílios com telefone seja inferior a cinquenta mil. Nesses casos, são esperados erros máximos de três pontos percentuais para a frequência de qualquer fator de risco na população adulta e de 
cerca de quatro pontos percentuais são esperados para estimativas específicas, segundo o sexo.

Seguindo os passos metodológicos adotados por Lima et al., (2017) ao analisarem a série histórica dos tipos principais de exercício físico e esporte recreativo praticados por adultos na cidade de Curitiba-PR, a variável de interesse central deste estudo foi o autorrelato de adultos residentes na cidade de São Luís - MA, sobre a escolha do tipo principal de exercício físico e esporte praticado no lazer. O inquérito do Vigitel oferece 16 opções de atividades, tendo o respondente, a possibilidade de apenas uma opção dentre: 1) caminhada; 2) caminhada em esteira rolante; 3) corrida; 4) corrida em esteira rolante; 5) musculação; 6) ginástica aeróbica; 7) hidroginástica; 8) ginástica geral; 9) natação; 10) artes marciais e lutas; 11) bicicleta (doravante nominada "ciclismo"); 12) futebol (desde o ano de 2016, foi adicionado a opção da modalidade de futsal); 13) basquetebol; 14) voleibol (desde o ano de 2016, foi adicionado a opção da modalidade de futvolei); 15) tênis; 16) Dança (balé, dança de salão, dança do ventre, foi introduzida como alternativa a partir do ano de 2013); 17) outros (soma de todas as modalidades citadas não nominadas de 1 a 16).

Para fins de análise, pesos amostrais foram considerados para os resultados serem extrapolados para toda a população adulta de São Luís. O método "rake" foi usado para estimar a população total de cada ano com base em dados censitários do ano correspondente. A análise descritiva determinou os valores centrais pela média aritmética e desvio padrão. A porcentagem da taxa de variação anual da série foi obtida mediante a modelagem de regressão de Prais-Winsten para os cinco tipos principais de exercício físico ou esporte praticado no tempo de lazer (variáveis dependentes) e o ano do inquérito (variável independente), para mensurar a variação da reta que ajusta os pontos da série temporal, o que propicia vantagens para a análise de regressão linear, como a redução da heterogeneidade de variância dos resíduos da análise de regressão. Devido às diferenças na escolha do tipo de exercícios e/ou esporte recreativo entre os sexos, observados em estudos prévios (LIMA et al., 2019), os dados foram analisados separadamente para homens e mulheres. Taxa de variação anual e intervalos de confiança de $95 \%$ foram calculados para cada variável. Mudanças significativas nos indicadores do tipo de exercício físico ou esporte foram indicadas quando os coeficientes da regressão foram significativamente diferentes de zero $(\mathrm{p}<0,05)$. Todas as análises foram realizadas no pacote estatístico Stata MP (ver. 11.0). 
O projeto Vigitel do período de 2006 a 2018 foi aprovado pela Comissão Nacional de Ética em Pesquisa para Seres Humanos do Ministério da Saúde. O banco de dados do Vigitel é público está disponível na Internet em: <http://svs.aids.gov.br/bases_vigitel_viva/vigitel.php> sem a possibilidade de identificação dos entrevistados. Desse modo, segundo a Resolução 510 - CNS, de 07 de abril de 2016, não serão registradas nem avaliadas pelo sistema CEP/CONEP as pesquisam que utilizem informações de domínio público cujas informações de banco de dados estejam agregadas, publicizadas e com impossibilidade de identificação do participante (BRASIL, 2016).

\section{RESULTADOS}

No período de 2006 a 2018, foram entrevistados pelo Vigitel, por via telefônica fixa, 24.412 adultos de ambos os sexos ( $\geq 18$ anos de idade), residentes na cidade de São Luís - MA (BRA). Do conjunto da amostra, 9.250 eram homens com média de idade de 41,4 $\pm 2,4$ anos e, 15.162 eram mulheres com média de idade de 43,9 $\pm 3,2$ anos. No período examinado, 11.440 (47\%) da amostra declarou praticar algum tipo de exercício físico ou esporte recreativo nos três últimos meses que antecederam a entrevista, sendo 5.383 homens e 6.057 mulheres. Após a aplicação de pesos amostrais individuais para sexo, a distribuição ponderada de ativos foi de $58,2 \%$ e $39,9 \%$, para homens e mulheres, respectivamente (Tabela 1 ).

Tabela 1 - Frequência absoluta de entrevistas completadas com adultos ( $\geq 18$ anos de idade) residentes na cidade de São Luís - MA, das mostras do Sistema de Vigilância de Fatores de Risco e Proteção para Doenças Crônicas por Inquérito Telefônico no período de 2006 a 2018.

\begin{tabular}{cccc}
\hline \multirow{2}{*}{ ANO } & Total (n) & Homens (n) & Mulheres (n) \\
& Média da idade - anos (DP) & Média da idade - anos (DP) & Média da idade - anos (DP) \\
2006 & 1223 & 444 & 779 \\
& $38,9(16,0)$ & $38,0(15,7)$ & $39,3(16,1)$ \\
2007 & 2012 & 776 & 1236 \\
& $40,2(15,9)$ & $39,4(15,8)$ & $40,6(15,9)$ \\
2008 & 2013 & 787 & 1226 \\
& $40,9(16,2)$ & $39,4(16,0)$ & $41,8(16,4)$ \\
2009 & 2011 & 794 & 1217 \\
& $40,9(16,0)$ & $39,9(15,8)$ & $41,5(16,1)$ \\
\hline
\end{tabular}




\begin{tabular}{cccc}
\hline \multirow{2}{*}{2010} & 2021 & 761 & 1260 \\
& $41,7(16,4)$ & $40,0(15,7)$ & $42,7(16,6)$ \\
2011 & 2009 & 782 & 1227 \\
& $42,8(16,6)$ & $41,6(16,9)$ & $43,6(16,3)$ \\
2012 & 1675 & 650 & 1025 \\
& $42,6(16,7)$ & $41,2(16,7)$ & $43,5(16,6)$ \\
2013 & 1942 & 713 & 1229 \\
& $44,8(17,7)$ & $42,4(18,1)$ & $46,2(17,3)$ \\
2014 & 1512 & 595 & 917 \\
& $44,8(16,8)$ & $43,0(16,0)$ & $46,0(17,3)$ \\
2015 & 2005 & 719 & 1286 \\
& $47,4(17,6)$ & $45,0(17,4)$ & $48,8(17,5)$ \\
2016 & 1934 & 752 & 1182 \\
& $47,8(17,9)$ & $45,4(18,2)$ & $49,3(17)$, \\
2017 & 2051 & 745 & 1306 \\
& $52,6(18,2)$ & $49,9(18,3)$ & $54,1(17,9)$ \\
2018 & 2004 & 732 & 1272 \\
& $52,5(18,1)$ & $49,6(18,3)$ & $54,2(17,8)$ \\
\hline
\end{tabular}

Fonte: Próprios autores.

DP - Desvio padrão da média da idade (anos).

A tabela 2 apresenta a frequência de adultos ( $\geq 18$ anos de idade) que relataram o tipo principal de exercício físico ou esporte praticado no tempo livre, no período de 2006 a 2018. Para o conjunto da amostra, os cinco principais modalidades foram: caminhada, futebol, musculação, ginástica em geral e corrida. Nesta série histórica, a caminhada foi destaque como a atividade mais referida, alcançando percentual médio de 19,3\% e, tendência de crescimento anual de $0,68 \%$. A prática de futebol recreativo ocupou o segundo lugar $(6,8 \%)$ e apresentou a maior taxa de decréscimo anual (-0,37\%). Na sequência, a musculação, com 6,1\% de preferência e crescimento anual de $0,22 \%$ ocupou a terceira posição, continuado pela ginástica e a corrida, com $2,2 \%$ e $2,0 \%$, respectivamente.

As tabelas 3 e 4 apresentam a distribuição da frequência relativa dos tipos principais de exercício físico ou esporte praticado no lazer por homens e mulheres, respectivamente. Entre os homens, os cinco principais tipos de esporte ou exercício físico mais referidos foram: caminhada, futebol, musculação, corrida e ciclismo. Entre as mulheres, foram: caminhada, musculação, ginástica em geral, caminhada em esteira e hidroginástica. Durante o período de acompanhamento, a caminhada foi a principal AF praticada por ambos os sexos. O percentual médio da escolha da caminhada foi de 17,7\% para homens e 20,3\% para mulheres, apresentando a maior tendência de crescimento anual, 0,68\% e 0,96\% para homens e 
mulheres, respectivamente. A prática de futebol recreativo se destacou como preferência masculina (17,3\%), enquanto para as mulheres, o futebol recreativo não alcançou meio ponto percentual. Contudo, foi a prática de futebol que apresentou a maior taxa de decréscimo anual $(-0,88 \%)$ entre o público masculino. A musculação ocupou o segundo lugar para mulheres $(5,4 \%)$ e a terceira posição para homens $(7,2 \%)$. A corrida $(3,7 \%)$ e o ciclismo $(3,1 \%)$ ocuparam o quarto e quinto lugar na preferência masculina e, a ginástica em geral $(2,8 \%)$, a caminhada em esteira $(1,9)$ e a hidroginástica $(1,8 \%)$ ocuparam o terceiro, quarto e quinto lugares, respectivamente, entre as mulheres. 
Tabela 2 - Percentual de adultos ( $\geq 18$ anos de idade) que relataram o tipo principal de exercício físico ou esporte praticado no lazer, segundo a cidade de São Luís, das amostras do Sistema de Vigilância de Fatores de Risco e Proteção para Doenças Crônicas por Inquérito Telefônico, no período de 2006 a 2018.

\begin{tabular}{|c|c|c|c|c|c|c|c|c|c|c|c|c|c|c|c|c|}
\hline Total & 2006 & 2007 & 2008 & 2009 & 2010 & 2011 & 2012 & 2013 & 2014 & 2015 & 2016 & 2017 & 2018 & Média & Coeficiente $^{5}$ & $\mathrm{p}$ \\
\hline \multicolumn{17}{|l|}{ Modalidades } \\
\hline Artes marciais e luta & & 0,2 & 0,1 & 0,3 & 0,2 & 0,2 & 0,5 & 0,5 & 0,6 & 0,6 & 1,1 & 1,0 & 0,6 & 0,5 & 0,074 & $<0,001$ \\
\hline Basquetebol & 0,3 & 0,3 & 0,2 & 0,2 & 0,2 & 0,2 & 0,1 & 0,1 & 0,1 & 0,1 & 0,2 & & 0,3 & 0,2 & $-0,010$ & 0,310 \\
\hline Ciclismo & 2,5 & 2,0 & 2,4 & 1,6 & 1,6 & 1,2 & 2,3 & 1,9 & 1,5 & 1,6 & 1,4 & 2,0 & 1,9 & 1,8 & $-0,040$ & 0,193 \\
\hline Caminhada $^{1}$ & 18,2 & 17,5 & 15,5 & 17,5 & 16,5 & 16,2 & 19,5 & 18,1 & 17,6 & 22,6 & 21,1 & 23,9 & 26,5 & 19,3 & 0,682 & 0,004 \\
\hline $\begin{array}{l}\text { Caminhada em } \\
\text { esteira }\end{array}$ & 0,8 & 0,9 & 1,2 & 1,0 & 1,3 & 1,8 & 1,9 & 2,2 & 2,1 & 0,8 & 2,1 & 2,2 & 1,0 & 1,5 & 0,069 & 0,087 \\
\hline Corrida & 1,0 & 1,0 & 1,5 & 1,4 & 1,5 & 1,8 & 2,0 & 1,8 & 2,2 & 2,3 & 3,3 & 2,8 & 3,1 & 2,0 & 0,178 & $<0,001$ \\
\hline Corrida em esteira & 0,3 & 0,1 & 0,2 & 0,1 & 0,1 & 0,5 & 0,0 & 0,4 & 0,3 & 0,1 & 0,5 & 0,4 & 0,4 & 0,3 & 0,020 & 0,008 \\
\hline $\operatorname{Dança~}^{2}$ & & & & & & & & 0,5 & 0,7 & 0,9 & 2,0 & 1,7 & 2,2 & 1,3 & 0,027 & 0,777 \\
\hline Futebol & 8,1 & 8,4 & 9,0 & 7,8 & 8,3 & 7,3 & 7,3 & 6,2 & 6,2 & 5,1 & 6,1 & 5,0 & 4,0 & 6,8 & $-0,367$ & $<0,001$ \\
\hline Ginástica aeróbica $^{3}$ & 0,5 & 0,9 & 0,6 & 0,8 & 0,8 & 1,0 & 1,0 & 0,7 & 1,7 & 1,1 & 1,2 & 1,1 & 0,9 & 1,0 & 0,046 & 0,007 \\
\hline Ginástica em geral ${ }^{4}$ & 1,1 & 1,6 & 1,0 & 1,3 & 1,4 & 1,2 & 2,1 & 2,5 & 3,3 & 2,4 & 2,9 & 3,2 & 4,2 & 2,2 & 0,246 & $<0,001$ \\
\hline Hidroginástica & 0,7 & 0,9 & 0,7 & 0,8 & 0,8 & 1,0 & 1,3 & 1,1 & 1,8 & 1,5 & 1,5 & 1,3 & 2,0 & 1,2 & 0,093 & $<0,001$ \\
\hline Musculação & 4,1 & 5,8 & 5,2 & 5,1 & 5,5 & 5,5 & 6,2 & 7,4 & 7,4 & 7,1 & 6,7 & 7,1 & 6,6 & 6,1 & 0,215 & 0,002 \\
\hline Natação & 0,3 & 0,8 & 0,7 & 0,3 & 0,2 & 0,5 & 0,6 & 0,8 & 0,3 & 0,4 & 0,6 & 0,5 & 0,5 & 0,5 & 0,001 & 0,962 \\
\hline Tênis & 0,2 & 0,1 & 0,1 & & 0,1 & 0,1 & 0,1 & 0,1 & 0,1 & & 0,1 & & 0,1 & 0,1 & $-0,005$ & 0,128 \\
\hline Voleibol & 0,6 & 0,5 & 0,1 & 0,5 & 0,6 & 0,1 & 0,1 & 0,4 & 0,3 & 0,2 & 0,5 & 0,3 & 0,3 & 0,4 & $-0,014$ & 0,256 \\
\hline
\end{tabular}

\footnotetext{
Fonte: Próprios autores.
}

${ }^{1}$ - não vale o deslocamento para trabalho; ${ }^{2}$ - a dança integrou o relatório do Vigitel somente a partir de 2013; ${ }^{3 i n c l u i ~ s p i n n i n g, ~ s t e p ~ e ~ j u m p ; ~}{ }^{4}$ - inclui alongamento, pilates e ioga. ${ }^{5}$ Coeficiente de Prais-Winsten. 
Tabela 3 - Percentual de adultos (18 anos ou mais de idade) do sexo masculino que relataram o tipo principal de exercício físico ou esporte praticado no lazer, segundo a cidade de São Luís, das amostras do Sistema de Vigilância de Fatores de Risco e Proteção para Doenças Crônicas por Inquérito Telefônico, no período de 2006 a 2018.

\begin{tabular}{|c|c|c|c|c|c|c|c|c|c|c|c|c|c|c|c|c|}
\hline Homens & 2006 & 2007 & 2008 & 2009 & 2010 & 2011 & 2012 & 2013 & 2014 & 2015 & 2016 & 2017 & 2018 & Média & Coeficiente $^{5}$ & $\mathbf{p}$ \\
\hline \multicolumn{17}{|l|}{ Modalidades } \\
\hline Artes marciais e luta & & 0,5 & 0,1 & 0,5 & 0,5 & 0,4 & 1,1 & 0,8 & 1,0 & 1,3 & 1,7 & 2,0 & 1,4 & 0,9 & 0,144 & $<0,001$ \\
\hline Basquetebol & 0,9 & 0,5 & 0,5 & 0,5 & 0,3 & 0,5 & 0,2 & 0,1 & & 0,4 & 0,4 & 0,1 & 0,8 & 0,4 & $-0,022$ & 0,266 \\
\hline Ciclismo & 5,0 & 3,2 & 3,3 & 2,4 & 3,2 & 2,0 & 3,4 & 3,2 & 2,7 & 2,2 & 2,4 & 3,8 & 3,4 & 3,1 & $-0,059$ & 0,342 \\
\hline Caminhada ${ }^{1}$ & 15,1 & 14,7 & 12,7 & 15,7 & 13,7 & 13,3 & 18,2 & 16,0 & 17,6 & 20,7 & 22,1 & 24,0 & 26,1 & 17,7 & 0,955 & 0,001 \\
\hline Caminhada em esteira & 2,3 & 0,5 & 0,8 & 0,6 & 0,8 & 1,0 & 1,7 & 2,7 & 1,0 & 0,8 & 1,5 & 1,7 & 1,4 & 1,3 & 0,032 & 0,543 \\
\hline Corrida & 0,7 & 2,3 & 3,2 & 2,8 & 3,0 & 3,1 & 4,2 & 3,4 & 4,4 & 4,0 & 5,6 & 5,8 & 6,4 & 3,7 & 0,371 & $<0,001$ \\
\hline Corrida em esteira & & 0,5 & 0,1 & 0,5 & 0,5 & 0,4 & 1,1 & 0,8 & 1,0 & 1,3 & 0,5 & 0,5 & 0,5 & 0,6 & 0,025 & 0,041 \\
\hline Dança $^{2}$ & & & & & & & & & 0,2 & 0,1 & 0,3 & 0,5 & 0,3 & 0,3 & 0,171 & 0,114 \\
\hline Futebol & 20,9 & 21,5 & 22,4 & 19,0 & 20,9 & 18,0 & 18,3 & 16,4 & 15,0 & 13,6 & 15,4 & 13,6 & 10,4 & 17,3 & $-0,882$ & $<0,001$ \\
\hline Ginástica aeróbica ${ }^{3}$ & & 0,3 & 0,3 & 0,4 & 0,1 & & 0,6 & 0,7 & 0,7 & 0,6 & 0,7 & 0,7 & 0,5 & 0,4 & 0,050 & 0,006 \\
\hline Ginástica em geral $^{4}$ & 0,9 & 1,3 & 1,3 & 1,4 & 0,7 & 1,7 & 0,5 & 2,0 & 1,8 & 1,4 & 1,7 & 1,2 & 1,1 & 1,3 & 0,031 & 0,209 \\
\hline Hidroginástica & & & 0,1 & 0,1 & 0,1 & 0,3 & 0,9 & 0,3 & & 0,4 & 0,1 & 0,4 & 0,7 & 0,3 & 0,037 & 0,068 \\
\hline Musculação & 6,5 & 6,7 & 7,4 & 6,0 & 5,4 & 7,7 & 6,6 & 8,8 & 9,6 & 8,6 & 6,3 & 7,9 & 6,7 & 7,2 & 0,100 & 0,337 \\
\hline Natação & 0,2 & 1,3 & 1,5 & 0,4 & 0,4 & 0,6 & 0,5 & 0,8 & 0,3 & 0,4 & 0,1 & 1,3 & 1,0 & 0,7 & 0,025 & 0,528 \\
\hline Tênis & 0,5 & 0,3 & 0,3 & 0,1 & 0,3 & 0,3 & 0,2 & 0,3 & & 0,1 & 0,7 & 0,1 & 0,3 & 0,3 & $-0,014$ & 0,034 \\
\hline Voleibol & 1,1 & 1,4 & 0,3 & 0,6 & 1,3 & 0,1 & & 0,8 & 0,5 & 0,7 & 0,7 & 0,7 & 0,7 & 0,7 & $-0,029$ & 0,326 \\
\hline
\end{tabular}

Fonte: Próprios autores

1 - não vale o deslocamento para trabalho; 2 - a dança integrou o relatório do Vigitel somente a partir de 2013; 3inclui spinning, step ejump; 4 - inclui alongamento, pilates e ioga. 5Coeficiente de Prais-Winsten. 
Tabela 4 - Percentual de adultos (18 anos ou mais de idade) do sexo feminino que relataram o tipo principal de exercício físico ou esporte praticado no lazer, segundo a cidade de São Luís, das amostras do Sistema de Vigilância de Fatores de Risco e Proteção para Doenças Crônicas por Inquérito Telefônico, no período de 2006 a 2018.

\begin{tabular}{|c|c|c|c|c|c|c|c|c|c|c|c|c|c|c|c|c|}
\hline Mulheres & 2006 & 2007 & 2008 & 2009 & 2010 & 2011 & 2012 & 2013 & 2014 & 2015 & 2016 & 2017 & 2018 & Média & Coeficiente $^{5}$ & $\mathrm{p}$ \\
\hline \multicolumn{17}{|l|}{ Modalidades } \\
\hline Artes marciais e luta & & 0,1 & 0,1 & 0,2 & 0,1 & 0,2 & 0,2 & 0,2 & 0,3 & 0,2 & 0,8 & 0,4 & 0,2 & 0,2 & 0,034 & 0,003 \\
\hline Basquetebol & & 0,2 & 0,1 & 0,1 & 0,1 & & & & 0,1 & & & & 0,1 & & $-0,006$ & 0,078 \\
\hline Caminhada $^{1}$ & 20,0 & 19,3 & 17,3 & 18,7 & 18,3 & 18,1 & 20,3 & 19,4 & 17,6 & 23,6 & 20,6 & 23,8 & 26,8 & 20,3 & 0,503 & 0,011 \\
\hline Caminhada em esteira & 0,3 & 1,2 & 1,5 & 1,2 & 4,9 & 2,3 & 2,0 & 2,0 & 2,7 & 0,9 & 2,5 & 2,5 & 0,9 & 1,9 & 0,051 & 0,571 \\
\hline Corrida & 1,2 & 0,2 & 0,5 & 0,5 & 0,6 & 0,9 & 0,7 & 0,8 & 0,8 & 1,3 & 1,9 & 1,1 & 1,2 & 0,9 & 0,071 & 0,016 \\
\hline $\operatorname{Dança}^{2}$ & & & & & & & & 0,8 & 1,0 & 1,3 & 3,0 & 2,3 & 3,3 & 1,7 & 0,049 & 0,608 \\
\hline Futebol & 0,8 & 0,2 & 0,5 & 0,5 & 0,6 & 0,4 & 0,4 & 0,2 & 0,5 & 0,4 & 0,2 & 0,2 & 0,3 & 0,4 & $-0,024$ & 0,027 \\
\hline Ginástica aeróbica $^{3}$ & 0,8 & 1,3 & 0,8 & 1,2 & 1,3 & 1,6 & 1,3 & 0,7 & 2,3 & 1,5 & 1,6 & 1,3 & 1,1 & 1,3 & 0,041 & 0,103 \\
\hline Ginástica em geral ${ }^{4}$ & 1,2 & 1,8 & 0,9 & 1,2 & 1,8 & 0,9 & 3,2 & 2,8 & 4,3 & 3,0 & 4,6 & 4,3 & 6,0 & 2,8 & 0,372 & $<0,001$ \\
\hline Hidroginástica & 1,0 & 1,5 & 1,1 & 1,3 & 1,3 & 1,5 & 1,6 & 1,6 & 2,9 & 2,1 & 2,4 & 1,8 & 2,8 & 1,8 & 0,126 & $<0,001$ \\
\hline Musculação & 2,7 & 5,3 & 3,8 & 4,4 & 5,6 & 4,1 & 6,0 & 6,5 & 6,0 & 6,3 & 6,7 & 6,7 & 6,4 & 5,4 & 0,269 & $<0,001$ \\
\hline
\end{tabular}

Fonte: Próprios autores

${ }^{1}$ - não vale o deslocamento para trabalho; ${ }^{2}$ - a dança integrou o relatório do Vigitel somente a partir de 2013; ${ }^{3}$ inclui spinning, step e jump; ${ }^{4}$ - inclui alongamento, pilates e ioga. ${ }^{5}$ Coeficiente de Prais-Winsten 
Gráfico 1 - Tendências temporais da frequência relativa dos cinco tipos de atividade física recreativa mais praticados por adultos ( $\geq 18$ anos de idade) de ambos os sexos, na cidade de São Luís - MA, das amostras do Sistema de Vigilância de Fatores de Risco e Proteção para Doenças Crônicas por Inquérito Telefônico, no período de 2006 a 2018.
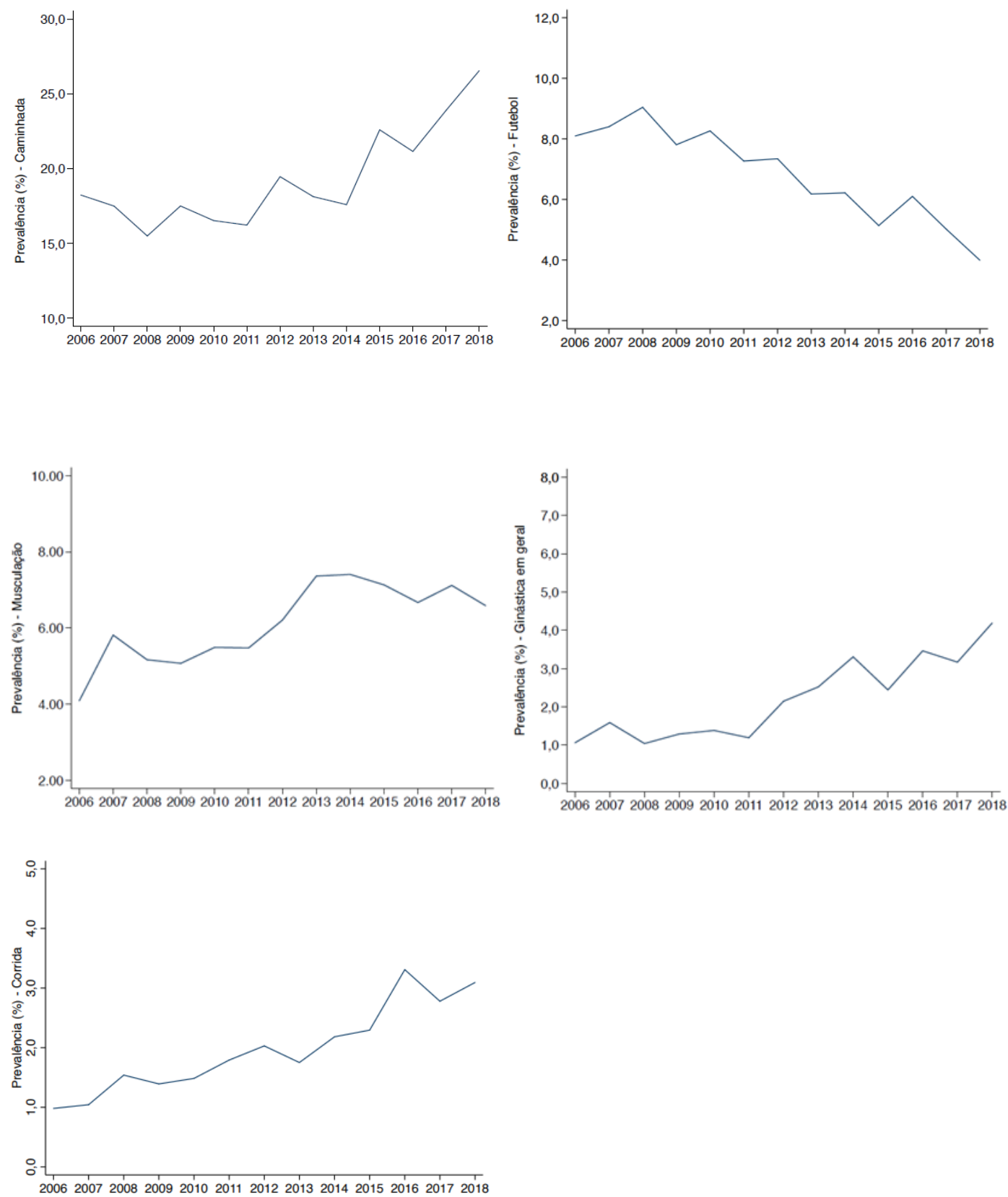

Fonte: Próprios autores. 


\section{DISCUSSÃo}

O sistema Vigitel, no período de 2006 a 2018, obteve amostras representativas da população adulta de ambos os sexos da cidade de São Luís - MA (BRA), possibilitando a análise dos tipos principais de exercício físico e esporte praticado no lazer na capital maranhense. A preferência pela caminhada se alinhou com os resultados do estudo de Lima et al. (2017) ao mostrarem a caminhada como a principal opção de AF de 17.184 adultos entrevistados por inquérito telefônico na cidade de Curitiba - PR, no período de 2006 a 2014. Também, com exceção da corrida e do futebol no estrato masculino, os demais resultados deste estudo se alinharam com os resultados encontrados por Cruz (2015), ao observar aumento da participação de AF aeróbia de intensidade moderada nas capitais brasileiras e no Distrito Federal no período de 2006 a 2013.

A caminhada como AF preferida foi mais prevalente entre as são-luisenses, no entanto, foram os homens que apresentaram a maior taxa de crescimento anual (0,96\%) para essa modalidade. Na eventual possibilidade dessa trajetória seguir inalterada, é possível projetar que a prevalência de marchadores na cidade de São Luís, a favor das mulheres, deixará de existir somente a partir do ano de 2050. Muito embora esse fenômeno requeira um modelo metodológico de estudo mais apropriado para ser explicado, é possível observar alinhamento da escolha da caminhada com a expectativa da taxa de envelhecimento da população brasileira (LIMA et al., 2018). A projeção da taxa de envelhecimento para a população de São Luís foi mostrada no gráfico 2. Desse modo, estes resultados parecem reafirmar que pessoas de maior idade tendem a escolher tipos de AF de intensidade menos vigorosa (CRUZ et al., 2018).

O melhor cenário esperado para o crescimento da taxa de marchadores na cidade de São Luís, seria aquele formado por um contingente de marchadores a partir do ingresso de pessoas inativas. De modo adicional, espera-se que as pessoas possam ser mais ativas no lazer à medida que se sintam mais seguras e protegidas no seu ambiente (LIMA et al., 2018) permitindo supor que a segurança urbana pode contribuir para dar robustez à taxa de crescimento dos marchadores. Dessa forma, a implementação de políticas urbanas encorajadoras à "caminhabilidade" deveria estabelecer estratégias consonantes e integradas com os setores da saúde, urbanismo, esporte, lazer, recreação e de segurança pública. 
Gráfico 2 - Projeção comparativa da distribuição da população brasileira e do estado do Maranhão para o ano de 2050, utilizando o ano de 2018 como referência. Para o ano de 2050, há uma projeção de que 1 em cada 4 brasileiros será idoso. O percentual de pessoas com mais de 65 anos passará dos atuais $9,2 \%$ para $25,5 \%$ (IBGE, 2019).

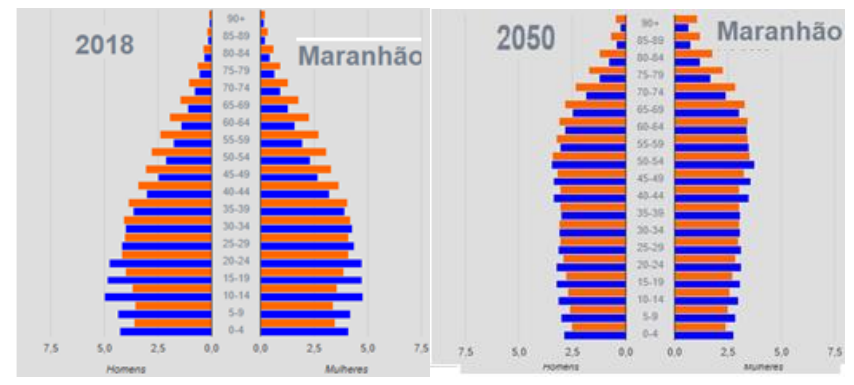

Fonte: IBGE (2019).

Mesmo o Brasil sendo conhecido popularmente como o "país do futebol”, há poucos estudos populacionais que descrevem detalhes da prática populacional do futebol recreativo. Um deles, (FLORINDO et al., 201), avaliou uma série histórica realizada no período de 2011 a 2015 , envolvendo 11.812 adultos ( $\geq 18$ anos de idade) praticantes de futebol recreativo. Nesse estudo, foi mostrado decréscimo de $1,4 \%$ ao ano da frequência de futebolistas e redução de 3,4\% para cada 5 anos a mais na idade deles. Outro estudo de 2006, analisou informações de 54.369 adultos entrevistados por inquérito telefônico, mostrando 17,6\% dos homens ativos praticando o futebol recreativo (LIMA et al., 2018). Na cidade de São Luís, a média da escolha pelo futebol recreativo, para o período investigado, foi de 17,3\%, acima da média nacional de $11,2 \%$. Os resultados reafirmaram ser o futebol um tipo de AF predominantemente masculina. Menos de meio ponto percentual de mulheres entrevistadas referiu o futebol como a principal forma de AF para se manterem ativas.

Um fator intrigante dos resultados deste estudo que merece destaque, foi a taxa de variação decrescente (- 0,89\% ao ano) para o futebol recreativo entre os homens. Esse valor se traduz em perda de $11 \%$ do número de praticantes de futebol recreativo na cidade de São Luís no período de 2006 a 2018. Projetando essa perda para valores absolutos, ao final do período estudado, foram encontrados, aproximadamente, quatro mil e quinhentos adultos (homens) a menos tendo o futebol recreativo como a sua principal AF de lazer. Essa variação negativa pode ser explicada, em parte, pela dificuldade de agrupar pessoas com interesses comuns e com condições físicas suficiente para praticar o futebol e, pela menor 
disponibilidade de espaços urbanos apropriados para a prática desta modalidade de AF, causada pela expansão imobiliária (FLORINDO et al., 2013).

O inquérito do Vigitel não permite saber se há deslocamento de futebolistas para outras modalidades de AF. Embora a explicação desse fenômeno não tenha sido o escopo desta investigação, ainda assim, parece coerente supor que haja migração. Esse pressuposto se sustenta na observação da estabilidade do número de inativos na cidade de São Luís (15,0\%) encontrada pelo Vigitel no período de 2014 a 2018 (Brasil, 2015; Brasil, 2017; Brasil, 2019) e pelo aumento das taxas de preferência para a caminhada, corrida e musculação (entre os ativos). Todavia, ao considerar essa suposição, é importante destacar que na investigação do Vigitel, fisicamente inativo foi considerado o adulto que não praticou qualquer AF no tempo livre nos últimos três meses, que não realizou esforços físicos intensos no trabalho, que não se deslocou para o trabalho ou curso/escola caminhando ou de bicicleta, perfazendo um mínimo de 20 minutos no percurso de ida e volta, e que não foi responsável pela limpeza pesada de sua casa.

A opção pela musculação como principal AF representou a escolha de 6,3\% do conjunto da amostra, sendo mais frequente em homens quando comparados às mulheres. Muito embora a taxa de crescimento anual da musculação em mulheres tenha sido três vezes maior à dos homens, se nada for feito e, a taxa de crescimento permanecer inalterada, serão necessários pelo menos 35 anos para a participação de mulheres se equiparar à de homens. 0 treinamento com exercícios resistidos (sinônimo de treinamento de força ou musculação) é fundamental para aumentar a força e manter a massa muscular. Nessa perspectiva, a OMS recomendada aos adultos realizarem musculação envolvendo os principais grupos musculares em dois ou mais dias na semana, de modo adicional às atividade aeróbias (150 minutos por semana de intensidade moderada e/ou 75 minutos de intensidade vigorosa). A revisão da literatura relacionando o trabalho de fortalecimento muscular à saúde pode ser acessada na revisão do Centros de Controle e Prevenção de Doenças dos Estados Unidos de 2008 (USDHHS, 2018) e nas revisões de Janssen (2007) e Janssen, Leblanc (2010).

A hidroginástica esteve presente entre as cinco atividades preferidas por mulheres. Alguns estudos recentes destacam vantagens para a prática de hidroginástica. Mostram que, dependendo da profundidade da imersão do corpo na água, o impacto articular e cardiocirculatório é menor no ambiente aquático quando comparado ao terrestre. Por 
exemplo, Kruel et al., (2001) observaram redução média da frequência cardíaca de 25 batimentos por minuto durante exercícios aquáticos com intensidade moderada. No entanto, algumas barreiras são limitadoras para a popularização dessa prática de AF. O reduzido espaço de locais apropriados (piscinas), a necessidade de cuidados de higiene dos praticantes e o cuidado com a qualidade da água das piscinas, geram custos financeiros que não podem deixar de estar previstos. Desse modo, parece não ser suficiente ofertar mais piscinas para aumentar a prática de hidroginástica, sendo necessário, também garantir as condições de uso.

A escolha do ciclismo apresentou taxas de variação anual estabilizadas para ambos os sexos, todavia, neste estudo, a frequência média de ciclista foi três vezes maior entre os homens. Os ciclistas, normalmente, apresentam como circunstâncias pessoais de pedalar, a promoção de saúde, o transporte ativo, a recreação e a aventura. Alguns estudos apontaram relação negativa do ciclismo como meio de transporte. Hardman et al., (2013), avaliaram 1.910 industriários e verificaram que somente $16 \%$ desses trabalhadores se deslocavam ativamente ao trabalho, a pé ou de bicicleta. No mesmo sentido, Silva et al., (2012), avaliaram 2.265 trabalhadores e mostraram $13,7 \%$ de homens e $12,8 \%$ de mulheres ativos no deslocamento para o trabalho. Desse modo, parece razoável supor, que a infraestrutura de acesso a equipamentos (bicicleta de locação), bicicletários e vias públicas seguras (ciclovias e ciclofaixas) pode estimular o uso recreativo de bicicleta e ampliar o emprego como meio de transporte. Essas ações poderiam somar significativamente em direção ao alcance das metas semanais de AF recomendadas.

A taxa de crescimento da escolha de ginástica apresentou crescimento positivo e foi alavancado principalmente pelo forte elevação nas mulheres. Os homens, principalmente os mais jovens, tendem a optar por atividades com intensidade mais vigorosa (LIMA et al., 2018). A participação em programas de ginástica guarda alguma semelhança com a participação em programas de musculação, ou seja, ambos se associam, de modo regular, à necessidade de contar com serviços especializados, cujo custo financeiro pode limitar o acesso ou a permanência nesses tipos de atividades.

Os motivos catalizadores de mudanças no nível de AF na população são-luisense, podem em parte, serem atribuídos a programas municipais de incentivo à promoção da AF. Como exemplos de intervenção local, cita-se a implantação do Projeto Conviver, proposto pelo governo municipal de São Luís para incentivar a prática da AF e desenvolver campanhas 
educativas para aquisição de hábitos saudáveis de vida voltadas ao público idoso, além da implantação do projeto São Luís Saudável, com objetivo de disponibilizar, gratuitamente, em espaços públicos, orientação de saúde e de qualidade de vida à população. De modo adicional, destaca-se a expansão de novas academias ao ar livre e a revitalização de aspectos paisagísticos do entorno dessas academias, requalificando os espaços urbanos na tentativa de atrair frequentadores. No entanto, como essas ações são desenvolvidas em locais fixos, muitas vezes inviabilizam a adesão e a aderência de usuários pelo distanciamento de suas moradias. Desse modo, tais aspectos relativos às mudanças de hábitos de vida necessitam ser melhor compreendidos em estudos específicos sobre o tema.

Limitações metodológicas devem ser consideradas neste estudo. A questão sobre o tipo de exercício físico ou esporte no Vigitel não é de múltipla escolha. Isso possibilita conhecer a preferência do tipo de AF praticado mas não possibilita conhecer as atividades complementares. Desse modo, um participante determina uma AF como principal, sem negar a participação em outra(s). Por isso, este estudo se limita a apresentar a principal escolha do entrevistado. Também, no Brasil, a cobertura da rede de telefonia não é homogênea, podendo ser menor em cidades economicamente menos desenvolvidas e nos estratos de menor nível socioeconômico. A utilização do uso de pesos pós-estratificados foi estabelecida para minimizar possíveis viesses da amostra.

Os pontos fortes deste estudo incluem informações do comportamento da participação em exercícios físicos e esporte de lazer da população adulta da cidade de São Luís e detalham movimentos temporais dessa participação no período de 2006 a 2016, especialmente para os profissionais da Educação Física e agentes interessados em planejar e promover a participação da população em atividades físicas recreativas. A análise da tendência de escolha por determinados tipos de exercício físico ou esporte na cidade de São Luís, reforça a necessidade de ações voltadas a assegurar o acesso e a manutenção para se efetivar essas escolhas.

\section{CONCLUSÕES}

A escolha da caminhada ao ar livre foi o tipo principal de AF recreativa relatado em ambos os sexos na cidade de São Luís, com tendência de crescimento positivo para ambos. 0 
futebol se confirmou como um esporte predominantemente masculino com taxas de variação decrescente ao longo do tempo. A taxa de variação anual do ciclismo permaneceu estável. A escolha de ginástica e de caminhada em esteira rolante cresceu significativamente entre as mulheres, enquanto a musculação apresentou crescimento positivo em ambos os sexos. Mudanças na escolha da AF no lazer na capital maranhense estão acontecendo e poderão ser monitorados mediante a continuidade e periodicidade do sistema Vigitel, que permitirá acompanhar os resultados das ações promotoras de AF. Finalmente, novos estudos podem definir as influências dos fatores sociodemográficos, econômicos e de comportamento individual para a escolha do tipo de atividade física mais adequada a cada grupo populacional, fornecendo informações para se estabelecer intervenções focais para o acompanhamento da adesão e aderência à prática de AF.

\section{REFERÊNCIAS}

BRASIL. Ministério da Saúde. Conselho Nacional de Saúde. Resolução no 510, de 7 de abril de 2016. Diário Oficial [da] República Federativa do Brasil, Brasília, DF, 24 maio 2016. Seção 1. p. 44-46. Disponível em: <http://conselho.saude.gov.br/resolucoes/2016/Reso510.pdf>. Acesso em: 13 agosto 2016.

Brasil. Ministério da Saúde. Secretaria de Vigilância em Saúde. Departamento de Vigilância de Doenças e Agravos não Transmissíveis e Promoção da Saúde. Vigitel Brasil 2017: vigilância de fatores de risco e proteção para doenças crônicas por inquérito telefônico. Ministério da Saúde, Secretaria de Vigilância em Saúde, Departamento de Vigilância de Doenças e Agravos não Transmissíveis e Promoção da Saúde. - Brasília: Ministério da Saúde, 2018. Disponível em: <https://bvsms.saude.gov.br/bvs/publicacoes/vigitel_brasil_2017_vigilancia_fatores_riscos.pdf>. Acesso em: 18 julho 2019.

BRASIL. Ministério da Saúde. Secretaria de Vigilância em Saúde. Departamento de Vigilância de Doenças e Agravos não Transmissíveis e Promoção da Saúde. Vigitel Brasil 2014: vigilância de fatores de risco e proteção para doenças crônicas por inquérito telefônico. Ministério da Saúde, Secretaria de Vigilância em Saúde, Departamento de Vigilância de Doenças e Agravos não Transmissíveis e Promoção da Saúde. - Brasília: Ministério da Saúde, $2015 . \quad$ Disponível <http://bvsms.saude.gov.br/bvs/publicacoes/vigitel_brasil_2014.pdf>. Acesso em: 14 setembro 2019.

BRASIL. Ministério da Saúde. Secretaria de Vigilância em Saúde. Departamento de Vigilância de Doenças e Agravos não Transmissíveis e Promoção da Saúde. Vigitel Brasil 2016: vigilância de fatores de risco e proteção para doenças crônicas por inquérito telefônico. Ministério da Saúde, Secretaria de Vigilância em Saúde, Departamento de Vigilância de Doenças e Agravos não Transmissíveis e Promoção da Saúde. - Brasília: Ministério da Saúde, 2017. Disponível em: <https://portalarquivos2.saude.gov.br/images/pdf/2018/marco/02/vigitel-brasil-2016.pdf\%3E>. Acesso em: 28 agosto 2019.

BRASIL. Ministério da Saúde. Secretaria de Vigilância em Saúde. Departamento de Vigilância de Doenças e Agravos não Transmissíveis e Promoção da Saúde. Vigitel Brasil 2018: vigilância de fatores de risco e proteção para doenças crônicas por inquérito telefônico. Ministério da Saúde, Secretaria de Vigilância em 
Saúde, Departamento de Vigilância de Doenças e Agravos não Transmissíveis e Promoção da Saúde. - Brasília: Ministério da Saúde, $2019 . \quad$ Disponível em: <https://portalarquivos2.saude.gov.br/images/pdf/2019/julho/25/vigitel-brasil-2018.pdf>. Acesso em: 28 agosto. 2019.

CRUZ, M. S. Tendência da prática de atividade física no lazer no Brasil (2006-2013). [Dissertação de Mestrado]. São Paulo: Faculdade de Saúde Pública da USP; 2015.Disponível em: <https://teses.usp.br/teses/disponiveis/6/6138/tde-11092015-113013/publico/MicheleSantosDaCruz.pdf>. Acesso em: 29 março 2019.

CRUZ, M. S.; BERNAL, R. T. I.; CLARO, R. M. Tendência da prática de atividade física no lazer entre adultos no Brasil (2006-2016). Cad. Saúde Pública, v. 34, n. 10, $2016 . \quad$ Disponível em:,<https://www.scielosp.org/article/csp/2018.v34n10/e00114817>. Acesso em: 30 junho de 2019.

LORINDO, A. A.; FARIAS JUNIOR, J. C.; WELLS, J. C. K.; HALLAL, P. C. Epidemiology of recreational football in Brazil: prevalence and association with risk factors for chronic diseases and self-rated health in adults. Motriz, v. 19, n. 1, p. 224-230, 2013. Disponível em: <https://www.scielo.br/pdf/motriz/v19n1/a23v19n1.pdf>. Acesso em: 16 janeiro 2019.

GUTHOLD, R.; STEVENS, G. A.; RILEY, L. M.; BUL, F. C. L. Worldwide trends in insufficient physical activity from 2001 to 2016: a pooled analysis of 358 population-based surveys with 1.9 million participants. The Lancet, v.6 (10), PE1077-E1086, 2018. Disponível em: <https://www.thelancet.com/journals/langlo/article/PIIS2214109X(18)30357-7/fulltext>. Acesso em: 20 agosto 2019.

HALLAL, P. C.; ANDERSEN, L. B.; BULL, F.C.; GUTHOLD, R.; HASKELL, W.; EKELUND, U. Global physical activity levels: surveillance progress, pitfalls and prospects. The Lancet, v.380, p. 247-257, 2012. Disponível em: <https://pubmed.ncbi.nlm.nih.gov/22818937/>. Acesso em: 14 agosto 2019.

HARDMAN, C. M.; BARROS, S. S. H.; OLIVEIRA, E. S. A.; NAHAS, M. V.; BARROS, M. V. G. Inatividade nos deslocamentos para o trabalho e fatores associados em industriários. Saúde Soc., v. 22, n. 3, p. 760-772, 2013. Disponível em: <https://www.scielosp.org/article/sausoc/2013.v22n3/760-772/>. Acesso em: 30 janeiro 2019.

INSTITUTO BRASILEIRO DE GEOGRAFIA E ESTATÍSTICA - IBGE. IBGE Cidades. Página oficial da Internet. Disponível em:<https://cidades.ibge.gov.br/brasil/ma/sao-luis/panorama>. Acesso em: 29 setembro 2018.

INSTITUTO BRASILEIRO DE GEOGRAFIA E ESTATÍSTICA [homepage na internet]. Projeção da população do Brasil e das Unidades da Federação. Disponível em: <https://www.ibge.gov.br/apps/populacao/projecao/>. Acesso em: 14 outubro 2019.

JANSSEN, I.; LEBLANC, A. Systematic Review of the Health Benefits of Physical Activity in School-Aged Children and Youth. International Journal of Behavioural Nutrition and Physical Activity, v. 7, n. 40, 2010. Disponível em:<https://pubmed.ncbi.nlm.nih.gov/20459784/>. Acesso em: 20 outubro 2019.

JANSSEN, I. Physical activity guidelines for children and youth. Applied Physiology Nutrition and Metabolism, v. 32, p. 109-121, 2007. Disponível em: <https://cdnsciencepub.com/doi/pdf/10.1139/H07-109>. Acesso em: 28 setembro 2019.

KOHL, H. W.; CRAIG, C. L.; LAMBERT, E. V.; INOUE, S.; ALKANDARI, J. R.; LEETONGIN, G.; KAHLMEIER, S. The pandemic of physical inactivity: global action for public health. The Lancet, v. 380, p. 294-305, 2012. Disponível em: <https://pubmed.ncbi.nlm.nih.gov/22818941/>. Acesso em: 10 julho 2109.

KRUEL, L. F. M.; MORAES, E. Z. C.; ÁVILA, A. O. V.; SAMPEDRO, R. M. F. Alterações fisiológicas e biomecânicas em indivíduos praticando exercícios de hidroginástica dentro e fora d'água. Revista Kinesis n. especial, p. 104-29, 2001. Disponível em: <https://periodicos.ufsm.br/kinesis/article/view/10373>. Acesso em: 15 fevereiro 2019.

LIMA, D. F, LIMA, L. A.; SILVA, M. P. Tendências temporais dos tipos principais de exercício físico e esporte praticados no lazer na cidade de Curitiba, Brasil: 2006- 2014. R. bras. Ci. e Mov, v. 25(3), p. 98-105, 2018. 
Disponível em: <https://portalrevistas.ucb.br/index.php/RBCM/article/view/98/275>. Acesso em: 20 fevereiro 2019.

LIMA, D. F.; ANGUERA, M. G.; LIMA, L. A.; JÚNIOR, O. M.; SAMPAIO, A. A.; JÚNIOR, A. P. S.; SILVA, M. P.; LUIZ, O. C. O Padrão Da Atividade Física De Brasileiros Diabéticos. Saúde (Santa Maria), v. 45, n. 2, p. 1-15, maio/agosto, 2019. Disponível em: <https://periodicos.ufsm.br/revistasaude/article/view/32659>.Acesso em: 27 agosto 2019.

LIMA, D. F.; LIMA, L. A.; MAZZARDO, O., ANGUERA, M. G.; PIOVANI, V.; JUNIOR, A. P. S.; SILVA, M.; SAMPAIO, A. A. $O$ padrão da atividade física no lazer de idosos brasileiros. Caderno de Educação Física e Esporte, v.6, n.2, p. 39-49. 2018. Disponível em: <http://e-revista.unioeste.br/index.php/cadernoedfisica/article/view/19111>. Acesso em: 16 agosto 2019.

LIMA, D. F.; PIOVANI, V. G. S.; LIMA, L. A. Recreational soccer practice among adults, in Brazilian capitals, 20112015. Epidemiol. Serv. Saude, v. 27, n. 2, p. e2017284, 2018. Disponível em: <https://pubmed.ncbi.nlm.nih.gov/29972472/>. Acesso em: 14 setembro 2019.

SILVA, S. G.; DUCA, G. F.; SILVA, K. S. S.; OLIVEIRA, E. S. A.; NAHAS, M. V. Deslocamento para o trabalho e fatores associados em industriários do sul do Brasil. Rev. Saúde Pública, v. 46, n. 1, p. 180-184, p. 2012. Disponível em: $<$ https://www.scielo.br/scielo.php?script=sci_arttext\&pid=\$0034-89102012000100022>. Acesso em: 10 fevereiro 2019.

US DEPARTMENT OF HEALTH AND HUMAN SERVICES. Physical Activity Guidelines for Americans. Office of Disease Prevention \& Health Promotion, Disponível em: <https://health.gov/our-work/physical-activity>. Acesso em 11 janeiro 2018.

WINTERS, M.; BRAUER, M.; SETTON, E. M.; TESCHKE, K. Built environment influences on healthy transportation choices: bicycling versus driving. J Urban Health, v. 87, n. 6, p. 969-93, 2010. Disponível em: $<$ https://pubmed.ncbi.nlm.nih.gov/21174189/>. Acesso em: 22 fevereiro 2019.

WORLD HEALTH ORGANIZATION (WHO). Global Action plan for the prevention and control of noncommunicable disease 2013-2020. Geneva, Switzerland: WHO, 2013.

WORLD HEALTH ORGANIZATION (WHO). Global recommendations on physical activity for health. Geneva, Switzerland: WHO, 2010.

WORLD HEALTH ORGANIZATION (WHO). Sustainable Development Goals (SDGs). Disponível em: <https://www.un.org/sustainabledevelopment/sustainable-development-goals/>. Acesso em: 29 maio 2019.

\section{(cc) $\mathbf{E Y}$}

Este trabalho está licenciado com uma Licença Creative Commons - Atribuição 4.0 Internacional. 